\title{
Diagnostic yield of rapid on-site evaluation transbronchial needle aspiration versus conventional transbronchial needle aspiration: a single center experience
}

\author{
Carmine Guarino ${ }^{1}$, Mariano Mollica ${ }^{2}$, Cristiano Cesaro ${ }^{1}$, Adriano Costigliola ${ }^{2}$, Maria Carolina Micheli ${ }^{3}$, \\ Nadia Corcione $^{2}$, Giuseppe La Cerra ${ }^{1}$, Carmelindo Tranfa ${ }^{2}$, Salvatore Cirillo ${ }^{2}$, Riccardo Smeraglio ${ }^{4}$, \\ Rosario Salvi ${ }^{5}$ \\ ${ }^{1}$ Bronchology Unit, Monaldi Hospital, A.O. dei Colli, Naples; ${ }^{2}$ Department of Translational Medical Sciences, \\ University of Campania "L. Vanvitelli", Monaldi Hospital, Naples; ${ }^{3}$ Pathology Unit, Monaldi Hospital, A.O. dei Colli, \\ Naples; ${ }^{4}$ Department of Public Health, University of Naples Federico II; ${ }^{5}$ Department of Thoracic Surgery, Monaldi \\ Hospital, A.O. dei Colli, Naples, Italy
}

\begin{abstract}
Trans-bronchial needle aspiration allows lymph node sampling in several thoracic conditions; the ability of Rapid On-Site Evaluation (ROSE) to predict the final diagnosis in this setting has
\end{abstract}

Correspondence: Mariano Mollica, Department of Translational Medical Sciences, University of Campania "L. Vanvitelli", Monaldi Hospital, via L. Bianchi, 80131 Naples, Italy.

Tel. +39.081.5453017 - Fax +39.081.7064135.

E-mail: mollicamariano@gmail.com

Key words: TBNA; bronchoscopy; Rapid On-Site Evaluation; NSCLC.

Conflict of interest: The Authors declare no conflict of interest with this manuscript.

Contribution: CG, FP, GG, CC, research design, study timetable planning; MM, CC, AC, NC, GLC, RS, SL, GP, patients' recruitment, data collection; NDR, GG, rapid on-site evaluation (ROSE) performing; FP, CG, CC, results analysis; RS, AC, NDR, FP, CG, CC, manuscript drafting. All the authors have read and approved the final version of the manuscript and agreed to be accountable for all aspects of the work.

Informed consent: obtained from all patients.

Ethics approval and consent to participate: All procedures performed in studies involving human participants were in accordance with the ethical standards of the Institutional Research Committee and with the 1964 Helsinki declaration and its later amendment.

Received for publication: 12 October 2019.

Accepted for publication: 3 January 2020.

${ }^{\circ}$ Copyright: the Author(s), 2020

Licensee PAGEPress, Italy

Monaldi Archives for Chest Disease 2020; 90:1144

doi: 10.4081/monaldi.2020.1144

This article is distributed under the terms of the Creative Commons Attribution Noncommercial License (by-nc 4.0) which permits any noncommercial use, distribution, and reproduction in any medium, provided the original author(s) and source are credited. not been well characterized. We performed a retrospective study to establish the utility of ROSE in the diagnosis of thoracic diseases with mediastinal lymph node involvement. We retrospectively reviewed 297 patients with hilar-mediastinal lymph node enlargement detected at CT scan from January 2013 to April 2016. 201 patients underwent conventional TBNA; in 96 patients, TBNA procedure was performed by on-site presence of a team of pathologists and research morphologists. Lung neoplasms, sarcoidosis, infections and lymphoma were the most common diseases diagnosed with TBNA samples. TBNA simultaneously performed in combination with ROSE produced an increase in percentage of appropriate samples compared to single cTBNA (adequate samples cTBNA $v s$ ROSE-TBNA: 73\% vs 81\%; p<0.05). Our observations indicate an increase in adequacy of fine needle aspirations and increased diagnostic yield in the ROSE group. In conclusions, ROSE may serve to reduce procedure time and enhance sample triaging therefore limiting the need for further invasive diagnostic testing.

\section{Introduction}

Lung cancer is the leading cause of cancer death worldwide; tobacco smoking and air pollution exposure are mainly implicated as risk factors [1-3]. Despite progresses in diagnostic procedures [4-9] lung cancer diagnosis occurs, in the majority of cases, when surgical approach is no longer feasible [10-12]. Lung cancer treatment has been renovated by target therapy and immune therapy [13-17] as a result of advances in understanding molecular basis of cancerogenesis [18-30]. This imply the need of adequate sampling procedures.

Trans Bronchial Needle Aspiration (TBNA) is a minimally invasive bronchoscopic technique which allows cytological, histological, or microbiological sampling of lesions within the airway wall, the lung parenchyma, and mediastinum adjacent to the tracheo-bronchial structures. Fine needles (usually 19 and 22 gauge) are introduced through the wall of the airway into a lymph node in order to obtain a specimen for cytological, histological or microbiological analysis. Hilar-mediastinal lymph node enlargement represent a major diagnostic challenge for both neoplastic and benign thoracic diseases [31-33]. TBNA is widely used procedure compared to more invasive mediastinoscopy and video-thora- 
coscopy for tissue specimen collection in lung cancer diagnosis and staging [34].

The diagnostic yield of TBNA may be influenced by several factors, including the operator experience, type of needle used, size and position of the lesion and prevalence of disease in the study population. Awareness of these factors, as highlighted by Bonifazi and colleagues [35], allows appropriate procedure planning which positively affects diagnostic accuracy.

The Rapid On-Site Evaluation (ROSE) is the intra-operative cytological evaluation of samples obtained by fine needle aspiration procedures, which has been shown to increase the diagnostic yield of samples (36). Even in absence of specific guidelines, a classification of the cytological samples obtained using TBNA has been proposed (Table 1). However, no general consensus on the definition of adequacy of trans-bronchial specimen examined with ROSE is so far available: some authors consider a sample adequate even with absence or low value of lymphocytes with presence of bronchial epithelial cells; other authors consider samples inadequate with all withdrawals $(\mathrm{C} 1, \mathrm{C} 3, \mathrm{C} 4)$ which do not allow a definitive diagnosis. Differing results have been reported in regard of the use of ROSE. In studies ranging from 1990 to 2010, which was mostly observational and not randomized, an increase of adequacy and/or diagnostic sensitivity of trans-bronchial samples assessed by the cytologist was reported. The randomized trials from 2011 to date, conducted by Trisolini [37] have shown that the ROSE was able to reduce the number of withdrawals for the diagnosis, without implications regarding the diagnostic yield, reducing the time of execution of the examination, and ultimately reducing the percentage of complications related to endoscopic maneuver (respiratory failure, bronchospasm, cardiac arrhythmias and hemodynamic instability, bleeding, fever). Baram and colleagues [38], have shown that a pre-procedural planning is able to predict the conditions in which the ROSE may have a better diagnostic yield. In fact, the ROSE may avoid additional biopsies to the patient where there is adequacy of the sample even in presence of multiple targets. Inadequate tissue sampling due to false positivity procedure represents the major risk for unsuccessful intra-operative cytological assessment: a close collaboration between endoscopist and pathologist may reduce this risk. As reported by Shannon et al. [39], the cTBNA + ROSE and EBUS-TBNA + ROSE methods seem to be overlapping in terms of diagnostic yield.

The costs related to the presence of the pathologist in endo- scopic room for ROSE was investigated by Bonifazi et al. [40] in comparison to the procedure performed by clinician trained by a short educational program of pulmonary pathology: the results indicate a procedural costs reduction. However, these studies were conducted in a single center with high-experience in cTBNA and EBUS-TBNA techniques, with established collaboration with pathologists. The impact of ROSE on diagnostic yield in a different setting has not been investigated.

Although the EBUS-TBNA, according to recent observations, is able to guarantee a greater diagnostic yield compared to cTBNA, there is still need to explore the effectiveness cTBNA as EBUS is not available in all centers due to high costs of the equipment and involvement a multidisciplinary team, including anesthetist. The aim of this study is to determine the impact of extemporaneous cytological evaluation (ROSE), in terms of adequacy of the sampling and diagnostic yield of TBNA on hilar or mediastinal lymph nodes detected by chest CT scan with contrast.

\section{Methods}

We retrospectively reviewed 297 patients, aged over 18 years, with hilar-mediastinal lymph nodes enlargement detected at CT scan (short axis $\geq 1 \mathrm{~cm}$ ) with or without detection of lung parenchymal consolidation admitted to Department of Cardio-Thoracic and Respiratory Sciences SUN at Monaldi Hospital, Naples, Italy, from January 2013 to April 2016. Written informed consent of the patients was systematically collected.

Two hundred and one patients underwent conventional TBNA; in 96 patients, TBNA procedure was performed by on-site presence of a team of pathologists and research morphologists. All TBNA sample were collected using a 22-gauge needle. The cytology slides were fixed and stained slides with fast dyes (Hemacolor Merck or Diff Quik). In Table 2 we have reported the characteristics of the two populations under examination, the two populations are similar.

Bronchoscopy was performed by using a routine bronchoscope (BFF260, 5.5-mm outer diameter of the tip; Olympus) in the presence of experienced respiratory physicians, cytotechnologist and nurses.

The vital signs of each patient were monitored during the pro-

Table 1. TBNA/ROSE specimens classification.

C1: sample not adequate or not evaluable

C2: benign outcome (normal cells, inflammatory disease)

C3: unclear outcome (cells with characteristics not completely attributable to benign disease)

C4: unclear outcome, probably malignant (cells with morphological atypia, in absence of specific features of malignancy)

C5: malignant outcome

Table 2. Main features in the two groups under examination.

\begin{tabular}{|c|c|c|}
\hline & TBNA group $(n=105)$ & TBNA + Rose group $(\mathrm{n}=96)$ \\
\hline Age & $63(55-71)$ & $65(53-69)$ \\
\hline Male & 41 & 39 \\
\hline Cardiovascular comorbidity & 30 & 37 \\
\hline Respiratory failure & 5 & 7 \\
\hline Sampled lymph nodes & $2(1-3)$ & $3(1-4)$ \\
\hline
\end{tabular}


cedure, including blood pressure, heart rate, respiratory rate, and oxygen saturation. The location of the bronchus leading to the lesion was planned on the basis of CT features. For the ROSE group, the materials from forceps biopsy imprint cytology were smeared onto glass slides. The glass slides were rapidly air-dried and stained immediately with a modified rapid Giemsa stain (DiffQuik stain). Microscopic examination of the slide preparation with Diff-Quik stain was performed within the bronchoscopy suite by two experienced cytotechnologists. In this manner, ROSE diagnosis was immediately communicated to the bronchoscopists. A cytotechnologist determined specimen adequacy.

Our diagnostic criterion for adequacy is the presence of cancer cells or cells suspicious for malignancy. The samples were classified as $\mathrm{C} 1$ to $\mathrm{C} 5$ according to the classification reported above. We considered a withdrawal as inadequate in presence of a small number of lymphocytes and prevalence of tracheobronchial epithelium cells. The sample was defined diagnostic if was within the $\mathrm{C} 2$ and $\mathrm{C} 5$ categories. If ROSE is positive, we will limit the sampling at this site to ensure the reliability of the results, reduce the risk of injury and bleeding. If ROSE is negative, we will repeat the biopsy. If all samples are negative according to the imaging prejudgment, and malignant disease is highly suspected clinically, we will recommend that patients undergo other tests, such as percutaneous puncture and thoracoscopic biopsy, in order to reduce the time until diagnosis. In each case, the remaining materials (ROSE group) or all specimens (non-ROSE group) were placed in 10\% formaldehyde and embedded in paraffin for routine histologic examination on hematoxylin and eosin staining. ROSE diagnoses were compared with the final pathologic diagnoses. The final diagnosis was based on the analysis of routine histologic pathology. The term malignant was defined as the presence of cancer cells or cells suspicious for malignancy. The samples were categorized as positive for malignancy or negative for malignancy, based on the results of ROSE. If flexible bronchoscopy produced a nonspecific diagnosis of malignancy, patients were referred for repeat flexible or rigid bronchoscopy, video-assisted thoracic surgery, or other surgical procedures. Data obtained were processed using the chi-square test.

\section{Results}

Of the 201 samples taken with conventional TBNA technique without on-site cytological evaluation, 55 samples were inadequate and 146 adequate (percentage of adequacy: 73\%). Of the 96 samples taken with ROSE + TBNA technique, 11 samples were inadequate and 85 were adequate (percentage of adequacy: $86.3 \%$ ) with $\mathrm{p}<0.05$ (Figure 1A). Figure 2 reports cytology assessment of lymph node specimens collected with ROSE-TBNA procedure. TBNA simultaneously performed in combination with ROSE produce an increase of percentage of appropriate samples compared to single cTBNA $(\mathrm{p}<0.05)$. In addition to the accuracy for histological diagnosis, ROSE in our study as exhibited a relevant favorable impact on adequacy of sample for molecular profiling. Of the 146 adequate cTBNA samples, a conclusive diagnosis (C2-C5) was achieved in 104 patients. The remaining 42 samples resulted in a non-definitive diagnosis. Among 85 adequate samples made with TBNA + ROSE technique, 70 patients obtained a conclusive diagnosis; 15 samples were not diagnostic results (C3-C4) (percentage of diagnostic success: $81 \%$ ) with $\mathrm{p}<0.05$. The most common diseases diagnosed which ROSE were primary tumor of the lung, sarcoidosis, TBC, lymphoma (Figure 1B). There were no false positive cases in the ROSE group. A second investigation level such as mediastinoscopy or video-assisted thoracoscopy (VATS) was required in patients with non-conclusive diagnosis. In addition to the impact of ROSE on diagnostic yield of TBNA sampling we have also explored the relevance of this methods on adequacy of samples for molecular profiling. We have considered adequate for a correct molecular profiling a samples exhibited at least $20 \%$ of tumor cell nuclei. According to this cut-off, 97\% of samples assessed with ROSE were adequate for molecular profiling.

\section{Discussion}

In this study, comparing a group of patients undergoing cTBNA with a group of patients undergoing TBNA + ROSE, we have demonstrated an increase in adequacy of fine needle aspirations and increased diagnostic yield in the group of patients where ROSE was performed. In addition, our observations highlight the importance of correct pre-operative planning in optimizing the endoscopic procedures and sampling collection in terms of assessment of number of lesions to be sampled, lymph node location, size of the lesions, selection needle type and sequence of sampling.

Conventional TBNA has been used for thirty years as a valuable tool in the diagnosis of hilar-mediastinal lymph nodes enlargement. However, according to the recent reports of the literature, the cTBNA and EBUS-TBNA guarantee a similar diagnostic yield when

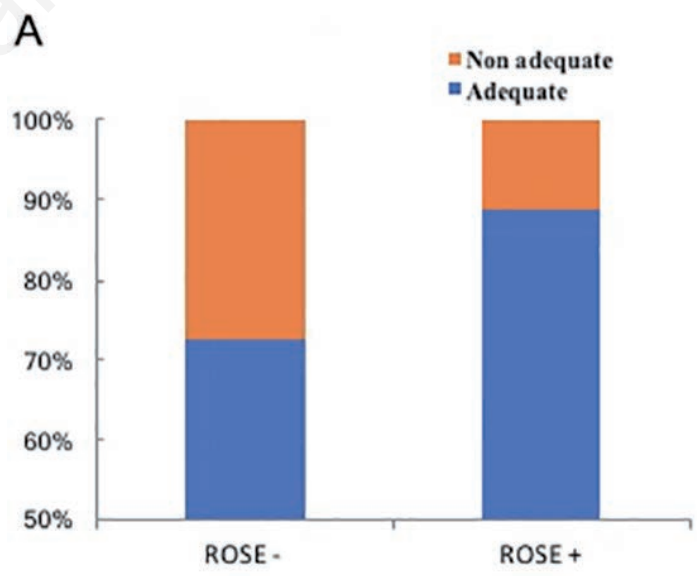

B

Diagnosis in adequate Rose+ samples

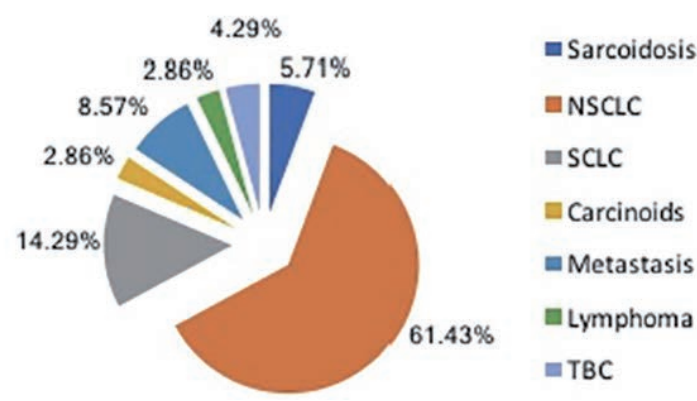

Figure 1. A) Graphic representation of the statistical analysis of the sample; procedures subjected to ROSE evaluation have a diagnostic yield significantly above $p<0.05$. B) Graphic representation of cytological results on TBNA samples. 

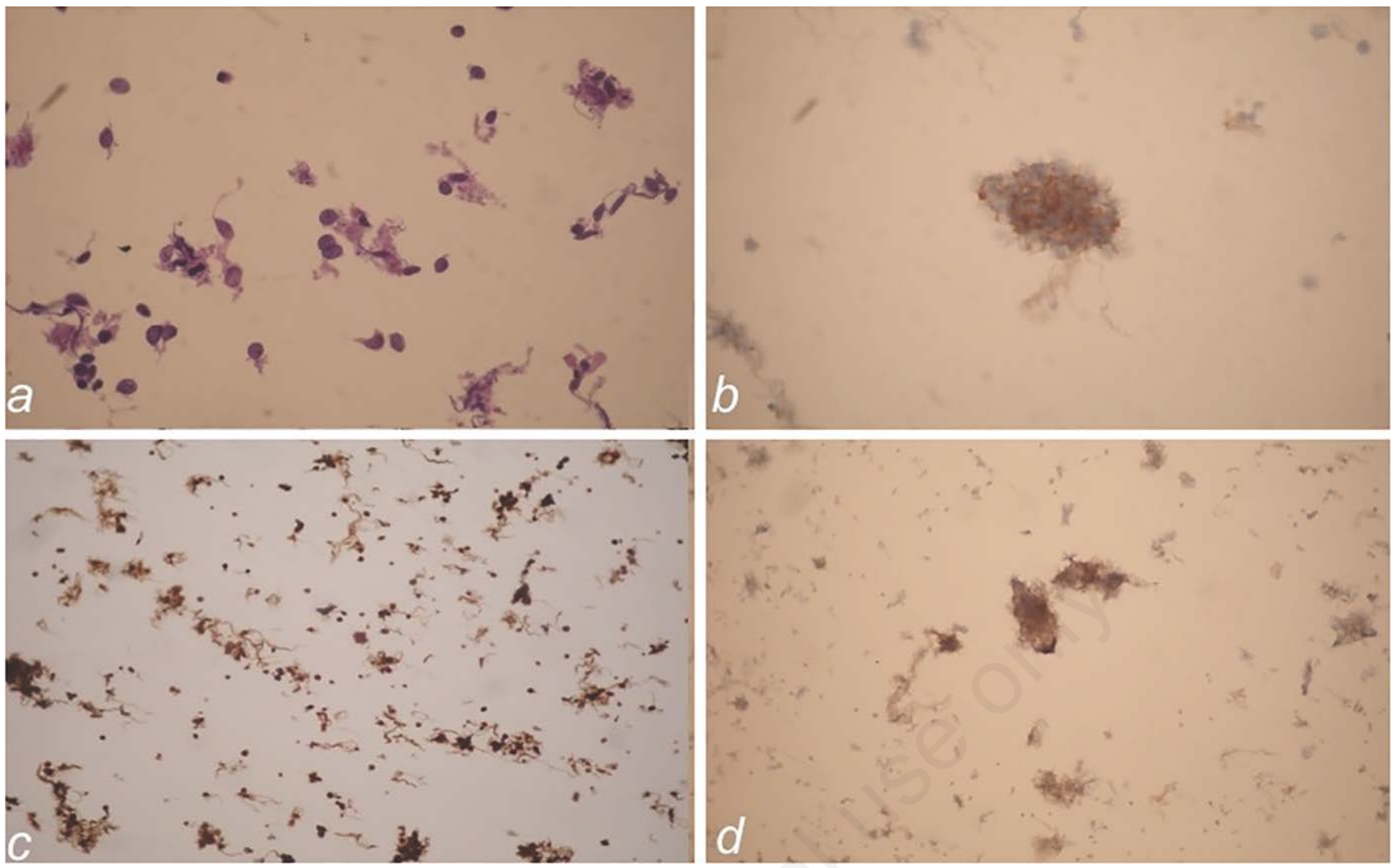

Figure 2. a,b) Lymph node TBNA sample: small mature lymphocytes with dense sheets of small cells with scant cytoplasm, finely granular nuclear chromatin. c) MIB-1 proliferation index. d) Positivity for synaptophysin.

used by experienced endoscopists. The role of ROSE of the samples obtained by trans-bronchial needle aspiration remains controversial. In a previous study, Shannon and colleagues [39] showed that the EBUS-TBNA have diagnostic performance comparable to TBNA + ROSE. In the present study, we have compared a group of patients undergoing TBNA with a group of patients undergoing cTBNA + ROSE; the results documented an increase in adequacy of fine needle aspirations and increased diagnostic yield in the ROSE group. Also, in our experience the correct pre-operative planning may optimize the procedure through the evaluation of several factors (number of lesions, lymph node location, size of the lesions, the needle type). The high cost EBUS still represents a limit to the wide spread of this technique. In this scenario, the ROSE can limit sampling bias, maximizing the diagnostic yield; the reduction of execution time of the procedure related to the ROSE also makes this technique a valid tool regarding patient outcomes. A multidisciplinary approach involving pulmonologist and pathologist during bronchial endoscopic procedures could therefore guarantee an accurate diagnosis even in absence of endoscopic ultrasound system.

\section{Conclusions}

The ROSE may optimize cTBNA procedure allowing a correct diagnosis in most of patients. The advantages of using this technique include reduction in the number of biopsies and average time and complications of the diagnostic procedure; also, the reduced use of sedative is preferable for the patient. In light of the growing need to obtain adequate tissue sampling for lung cancer diagnosis, the ROSE procedure can act, in absence of the EBUS, as "navigator" for the endoscopist, minimizing the risk of non-diagnostic procedure. In the era of precision medicine, the ROSE could be a relevant diagnostic tool to achieve accurate tissue sampling even for molecular biology investigations.

\section{References}

1. Turner MC, Cohen A, Jerrett M, et al. Interactions between cigarette smoking and fine particulate matter in the risk of lung cancer mortality in cancer prevention study II. Am J Epidemiol 2014;180:1145-9.

2. De Laurentiis G, Paris D, Melck D, et al. Separating smokingrelated diseases using NMR-based metabolomics of exhaled breath condensate. J Proteome Res Mar 2013;120:1502-11.

3. Mazzarella G, Lucariello A, Bianco A, et al. Exposure to submicron particles (PM1.0) from diesel exhaust and pollen allergens of human lung epithelial cells induces morphological changes of mitochondria tonifilaments and rough endoplasmic reticulum. In Vivo 2014;28:557-61.

4. Brunese L, Greco B, Setola FR, et al. Non-small cell lung cancer evaluated with quantitative contrast-enhanced CT and PET-CT: Net enhancement and standardized uptake values are related to tumour size and histology. Med Sci Monit 2013;19:95-101. 
5. Rinaldi L, Milione S, Fascione MC, et al. Relevance of ultrasound in the diagnostic algorithm of respiratory diseases in a real life setting: a multicenter prospective study. Respirology 2019. doi: 10.1111/resp.13659.

6. De Blasio F, Di Gregorio A, De Blasio F, et al. Malnutrition and sarcopenia assessment in patients with chronic obstructive pulmonary disease according to international diagnostic criteria, and evaluation of raw BIA variables. Respir Med 2018;134:1-5.

7. De Blasio F, de Blasio F, Miracco Berlingieri G, et al. Evaluation of body composition in COPD patients using multifrequency bioelectrical impedance analysis. Int $\mathrm{J}$ Chron Obstruct Pulmon Dis 2016;11:2419-26.

8. Maniscalco M, Bianco A, Mazzarella G, et al. Recent advances on nitric oxide in the upper airways. Curr Med Chem 2016;23:2736-45.

9. Maniscalco M, Vitale C, Vatrella A, et al. Fractional exhaled nitric oxide-measuring devices: technology update. Med Devices (Auckl) 2016;23;9:151-60.

10. Mazzella A, Izzo A, Amore D, et al. Single port VATS resection of a sessile solitary fibrous tumour of the visceral pleura. A case report. Ann Ital Chir 2015;86 (ePub).

11. Mollica M, Maffucci R, Lavoretano S, et al. Non-small cell lung cancer presenting as "psoas muscle syndrome". Monaldi Arch Chest Dis 2019;89:1012.

12. Mollica M, Salvi R, Paoli G, et al. Lung cancer management: challenges in elderly patients. J Gerontol Geriatr 2019;67:132-40.

13. Bianco A, Perrotta F, Barra G, et al. Prognostic factors and biomarkers of responses to immune checkpoint inhibitors in lung cancer. Int J Mol Sci 2019;20. pii: E4931. doi: 10.3390/ijms20194931.

14. Perrotta F, Rocco D, Vitiello F, et al. Immune checkpoint blockade for advanced NSCLC: A new landscape for elderly patients. Int J Mol Sci 2019;20. pii: E2258. doi: 10.3390/ijms20092258.

15. Fiorelli A, Perrotta F, Mollica M, et al. Endoscopic central airway recanalization to enable first line pembrolizumab treatment in a PD-L1 strongly positive non-small cell lung cancer: a case report. J Cardiothorac Surg 2019;14:50.

16. Bianco A, Campbell SFM. Atezolizumab plus platinum-based $r$ egimen and bevacizumab: Is it time to consider immunotherapy in a concurrent approach for lung cancer? Transl Cancer Res 2019;8:S103-5.

17. Bianco A, Malapelle U, Rocco D. et al. Targeting immune checkpoints in non small cell lung cancer. Curr Opin Pharmacol 2018;40:46-50.

18. Cattaneo F, Guerra G, Parisi M, et al. Expression of formylpeptide receptors in human lung carcinoma. Anticancer Res 2015;35:2769-74.

19. Nigro E, Stiuso P, Matera MG, et al. The anti-proliferative effects of adiponectin on human lung adenocarcinoma A549cells and oxidative stress involvement. Pulm Pharmacol Ther 2019;55:25-30.

20. Di Zazzo E, Polito R. Bartollino S, et al. Adiponectin as link factor between adipose tissue and cancer. Int J Mol Sci 2019;20:839.

21. Nigro E, Scudiero O, Monaco ML, et al. New insight into adiponectin role in obesity and obesity-related diseases. Biomed Res Int 2014;2014:658913.

22. Nigro E, Scudiero O, Sarnataro D, et al. Adiponectin affects lung epithelial A549 cell viability counteracting TNFa and IL$1 \beta$ toxicity through AdipoR1. Int $\mathrm{J}$ Biochem Cell Biol 2013;45:1145-53.

23. Nigro E, Imperlini E, Scudiero O, et al. Differentially expressed and activated proteins associated with non small cell lung cancer tissues. Respir Res 2015;16:74,

24. Cardarella S, Johnson BE. The impact of genomic changes on treatment of lung cancer. Am J Respir Crit Care Med 2013;188: 70-5.

25. Pilyugin M, Descloux P, André PA, et al. BARD1 serum autoantibodies for the detection of lung cancer. PLoS One 2017; 12:e0182356.

26. Illiano M, Nigro E, Sapio L, et al. Adiponectin down-regulates CREB and inhibits proliferation of A549 lung cancer cells. Pulm Pharmacol Ther 2017;45:114-20.

27. Bianco A, Nigro E, Monaco ML, et al. The burden of obesity in asthma and COPD: Role of adiponectin. Pulm Pharmacol Ther 2017;43:20-5.

28. Bianco A, Valente T, De Rimini ML, et al. Clinical diagnosis of malignant pleural mesothelioma. $J$ Thorac Dis 2018;10:S253-61.

29. Wu JY, Vlastos AT, Pelte MF, et al. Aberrant expression of BARD1 in breast and ovarian cancers with poor prognosis. Int J Cancer 2006;118:1215-26.

30. Zhang YQ, Bianco A, Malkinson AM, et al. BARD1: An independent predictor of survival in non-small cell lung cancer. Int J Cancer 2012;131:83-94.

31. Perrotta F, Cerqua FS, Cammarata A, et al. Integrated therapeutic approach to giant solitary fibrous tumor of the pleura: Report of a case and review of the literature. Open Med (Wars) 2016;11:220-5.

32. Punamiya V, Mehta A, Chhajed PN, et al. Bronchoscopic needle aspiration in the diagnosis of mediastinal lymphadenopathy and staging of lung cancer. J Cancer Res Ther 2010;6:134-41.

33. Guarino C, Mazzarella G, De Rosa N, et al. Pre-surgical bronchoscopic treatment for typical endobronchial carcinoids. Int J Surg 2016;33:S30-5.

34. Fiorelli A, Mazzella A, Pierdiluca M, et al. Routine invasive mediastinal staging of lung cancer in elderly patients without lymph adenopathy on PET-CT scan: is an appropriate choice? J Gerontol Geriatr 2017;65:18-37.

35. Bonifazi M, Sediari M, Ferretti M, et al. The role of the pulmonologist in rapid on-site cytologic evaluation of transbronchial needle aspiration: a prospective study. Chest 2014;145:60-5.

36. Cardoso AV, Neves I, Magalhaes A, et al. The value of rapid on-site evaluation during EBUS-TBNA. Rev Port Pneumol 2006;21:253-8.

37. Trisolini R, Cancellieri A, Tinelli C, et al. Rapid on-site evaluation of transbronchial aspirates in the diagnosis of hilar and mediastinaladenopathy: a randomized trial. Chest 2011;139: 395-01.

38. Baram D, Garcia RB, Richman PS. Impact of rapid on-site cytologic evaluation during transbronchial needle aspiration. Chest 2005;128:869-75.

39. Shannon JJ, Bude RO, Orens JB, et al. Endobronchial ultrasound-guided needle aspiration of mediastinaladenopathy. Am J RespirCrit Care Med 1996;153:1424-30.

40. Bonifazi M, Zuccatosta L, Trisolini R, et al. Transbronchial needle aspiration: a systematic review on predictors of a successful aspirate. Respiration 2013;86:123-34. 